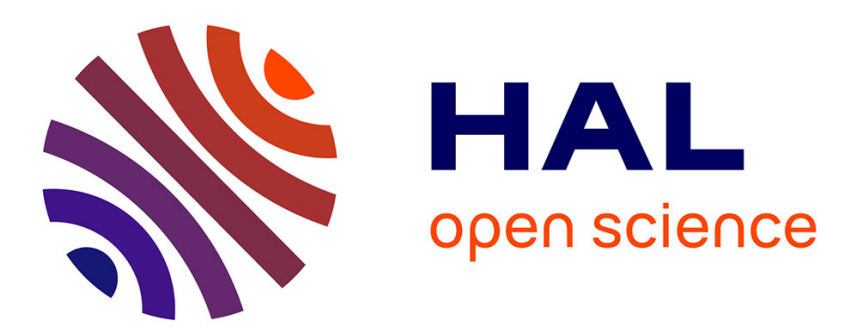

\title{
Electric field induced tricritical point in chiral polarized liquid crystals
}

\author{
A. Michelson, D. Cabib
}

\section{To cite this version:}

A. Michelson, D. Cabib. Electric field induced tricritical point in chiral polarized liquid crystals. Journal de Physique Lettres, 1977, 38 (15), pp.321-323. 10.1051/jphyslet:019770038015032100 . jpa00231386

\section{HAL Id: jpa-00231386 https://hal.science/jpa-00231386}

Submitted on 1 Jan 1977

HAL is a multi-disciplinary open access archive for the deposit and dissemination of scientific research documents, whether they are published or not. The documents may come from teaching and research institutions in France or abroad, or from public or private research centers.
L'archive ouverte pluridisciplinaire HAL, est destinée au dépôt et à la diffusion de documents scientifiques de niveau recherche, publiés ou non, émanant des établissements d'enseignement et de recherche français ou étrangers, des laboratoires publics ou privés. 


\title{
ELECTRIC FIELD INDUCED TRICRITICAL POINT IN CHIRAL POLARIZED LIQUID CRYSTALS
}

\author{
A. MICHELSON \\ and \\ D. CABIB \\ Physics Department, Technion, Haifa, Israel
}

(Reçu le 1 ${ }^{\mathrm{er}}$ juin 1977, accepté le 23 juin 1977)

\begin{abstract}
Résumé. - On présente une théorie de la transition entre un smectique $\mathrm{C}$ polarisé uniformément et un smectique $C^{*}$ hélicoïdal en présence d'un champ électrique parallèle aux couches, en utilisant la théorie de Landau. Le diagramme de phase (champ électrique-température) a un point tricritique $\left(E_{\mathrm{t}}, T_{\mathrm{t}}\right)$. Les valeurs de $E_{\mathrm{t}}$ et $T_{\mathrm{t}}$ sont exprimées en fonction des propriétés macroscopiques du matériau.

Abstract. - We present a Landau theory of the transition between a uniformly polarized smectic $\mathrm{C}$ phase and a distorted smectic $\mathrm{C}^{*}$ phase in the presence of an electric field parallel to the layers. The field-temperature $(E-T)$ phase diagram is shown to exhibit a tricritical point $\left(E_{\mathrm{t}}, T_{\mathrm{t}}\right)$. The values of $E_{\mathrm{t}}, T_{\mathrm{t}}$ are expressed in terms of observable macroscopic properties of the material.
\end{abstract}

We have been attempting recently [1] to explain some macroscopic properties of recently discovered [2-5] polarized liquid crystals composed of chiral molecules (DOBAMBC and related materials). These materials present a helicoidal smectic $\mathrm{C}$ phase (named the $\mathrm{C}^{*}$ phase) with spontaneously polarized layers. On the basis of the suggested [2] linear coupling between the layer polarization and the quadrupolar order parameter characterizing the tilt in the $C^{*}$ phase, we found qualitative agreement with experiments on the structure of the $C^{*}$ phase and on the linear response to an external electric field $E$ parallel to the layers. For $E=0$, experiments [2] and theory [1,6] agree in showing a second-order phase transition from the smectic A to the $\mathrm{C}^{*}$ phase. For an infinitesimal $E \neq 0$, the theory [1] predicts a monotonically decreasing line of critical temperatures $T_{\mathrm{c}}(E)$ separating a uniformly polarized smectic $\mathrm{C}$ phase (at $T>T_{\mathrm{c}}$ ) and a distorted smectic $\mathrm{C}^{*}$ phase (at $T<T_{\mathrm{c}}$ ). The distortion induced by the field in the $\mathrm{C}^{*}$ phase is essentially a tilt of the axis of the helix with respect to the layer normal, which makes the $\mathrm{C}^{*}$ phase biaxial.

In this paper, we go a step further in studying the line $T_{\mathrm{c}}(E)$ and show that it terminates at a tricritical point $\left(E_{\mathrm{t}}, T_{\mathrm{t}}\right)$, so that at $E>E_{\mathrm{t}}$ the phase transition becomes first order. As in the previous works [1,7], we use a formalism based on the Landau theory of second-order phase transitions.
Because of the linear coupling between the layer polarization and the tilt angle, these two are proportional below the transition [1, 2]. It can be shown [1] that only one complex parameter is independent, and one can choose $P=P_{x}+i P_{y}$ as such a parameter $\left(P_{x}, P_{y}\right.$ are the cartesian components of the layer polarization ; the $z$-axis is chosen to be normal to the layers). In the smectic $\mathrm{C}^{*}$ phase, $P$ varies helicoidally in the $z$-direction, with a pitch much larger than the interlayer distance. Therefore we will consider $P$ as a slowly varying function of $z$ and expand the free energy $F$ in both $P$ and $\partial P / \partial z$. The expansion of $F$ (per unit volume) has the form [7]

$$
\begin{gathered}
F=V^{-1} \int \frac{1}{2}\left[K_{1}|P|^{2}+\right. \\
+i K_{2}\left(P \partial P^{*} / \partial z-P^{*} \partial P / \partial z\right)+K_{3}|\partial P / \partial z|^{2}+ \\
\left.+K_{4}|P|^{4}-E\left(P+P^{*}\right)\right] \mathrm{d}^{3} r
\end{gathered}
$$

The integration is performed over the sample volume $V$, and the main assumption is that only $K_{1}$ is temperature dependent among the coefficients $K_{1}, \ldots, K_{4}$. The ordinary dielectric coupling, $-\frac{1}{2} \chi E^{2}$, is omitted from (1), since it is small [2] compared with the ferroelectric coupling, $-P E$. Expanding $P$ in a Fourier series $P(z)=\sum_{k} P_{k} \mathrm{e}^{i k z}$ and substituting 
into (1), we obtain

$$
\begin{aligned}
& \begin{aligned}
F=\frac{1}{2}\left[K_{1}\left|P_{0}\right|^{2}\right. & \left.+K_{4}\left|P_{0}\right|^{4}-E\left(P_{0}+P_{0}^{*}\right)\right]+ \\
& +\frac{1}{2} \sum_{k}^{\prime}\left[\left(A_{k}+4 K_{4}\left|P_{0}\right|^{2}\right)\left|P_{k}\right|^{2}\right. \\
& \left.+K_{4}\left(P_{0}^{* 2} P_{k} P_{-k}+\text { c.c. }\right)\right] \\
& +\frac{1}{2} K_{4} \sum_{k k^{\prime} k^{\prime \prime}}^{\Gamma^{\prime}} P_{k} P_{k^{\prime}} P_{k^{\prime \prime}}^{*} P_{k+k^{\prime}-k^{\prime \prime}}^{*},
\end{aligned} \\
& \text { where } \quad K_{1}+2 K_{2} k+K_{3} k^{2},
\end{aligned}
$$

and the primes exclude $k=0$ from the summation on $k$. The quantity $P_{0}$, corresponding to a uniform polarization, obviously vanishes for $E=0$. In this case the helicoidal phase is characterized by a pitch $l=2 \pi /\left|k_{0}\right|$, where $k_{0}$, the wave number of the soft mode of the smectic $A$ to $C^{*}$ phase transition, is determined from the minimization of $A_{k}: \partial A_{k} / \partial k=0$. This yields

$$
k_{0}=-K_{2} / K_{3} \text {. }
$$

The critical temperature $T_{\mathrm{c} 0}$ at $E=0$ is determined from the equation $A_{k_{0}}\left(T_{\mathrm{c}}\right)=0$, where

$$
A_{k_{0}}(T)=K_{1}(T)-K_{2}^{2} / K_{3} .
$$

Assuming linear dependence of $A_{k_{0}}$ on $T$ near $T_{\mathrm{co}}: A_{k_{0}}(T)=C^{-1}\left(T-T_{\mathrm{co}}\right)$, we obtain from (5) :

$$
K_{1}(T)=C^{-1}\left(T-T_{\mathrm{co}}\right)+K_{2}^{2} / K_{3} .
$$

The zero-field dielectric susceptibility at $T>T_{\mathrm{c} 0}$ is $\chi=K_{1}^{-1} ;$ it tends to the finite value

$$
\chi_{\mathrm{c}}=K_{3} / K_{2}^{2}
$$

at $T \rightarrow T_{\mathrm{co}}+0$, and behaves according to the CurieWeiss law

at

$$
\chi \cong C\left(T-T_{\mathrm{co}}\right)^{-1}
$$

$$
T-T_{\mathrm{c} 0} \gg C \chi_{\mathrm{c}}^{-1} \text {. }
$$

For $E \neq 0$, the higher-temperature phase is a uniformly polarized smectic $\mathrm{C}$, characterized by a real $P_{0} \neq 0$ and $P_{k}=0$ for all $k \neq 0 ; P_{0}$ is determined, as a function of $T$ and $E$, from the equation

$$
K_{1}(T) P_{0}+2 K_{4} P_{0}^{3}=E
$$

following from the minimization of $F$ with respect to $P_{0}$. As $T$ goes below $T_{\mathrm{c}}=T_{\mathrm{c}}(E)$, the free energy (2) becomes unstable with respect to the appearance of a non-zero $P_{k}$, in addition to $P_{0}$, so that a second-order transition occurs to a distorted (distorted because of $P_{0} \neq 0$ ) smectic $C^{*}$ phase. To determine the soft mode of this transition, one has to diagonalize the harmonic part of $F$, quadratic in $P_{k}(k \neq 0)$, and find the lowest eigenvalue $A_{k_{0}}^{\prime}$ of the quadratic expression. One thereby determines the wave number $k_{0}$ of the soft mode $\psi$ which is a certain linear combination of $\boldsymbol{P}_{\boldsymbol{k}_{0}}$ and $P_{-k_{0}}^{*}: \psi=\alpha P_{k_{0}}+\beta P_{-k_{0}}^{*} \cdot T_{\mathrm{c}}=T_{\mathrm{c}}(E)$ is found from the equation $A_{k_{0}}^{\prime}=0$ together with eq. (9). It is then possible to present $F$ in the effective form

$$
F=F_{0}+\frac{1}{2}\left(A_{k_{0}}^{\prime}|\psi|^{2}+B|\psi|^{4}+\cdots\right) .
$$

Then it can be shown that, on increasing $E$, the coefficient $B$ decreases along the line $T_{\mathrm{c}}(E)$, until it vanishes at the tricritical point $\left(E_{\mathrm{t}}, T_{\mathrm{t}}\right)$. The procedure described, which is simple but rather tedious, makes it possible to find the exact (within the Landau theory) parametric equations of the critical line $T_{c}(E)$ and the values of $E_{\mathrm{t}}, T_{\mathrm{t}}$ (expressed in terms of $\chi_{\mathrm{c}}, C$, and $K_{4}$ ). It turns out, however, that a much simpler, although approximate, calculation based on neglecting the off-diagonal terms $P_{k} P_{-k}$ in (2) leads to the same results with small differences (e.g., a difference of $4 \%$ in the value of $\left.E_{t}\right)$. For the sake of brevity and simplicity, we will use the approximate method here.

By neglecting the coupling terms $P_{k} P_{-k}$ in (2), the soft mode is again $\psi=P_{k_{0}}$ with $k_{0}$ from (4). However, $T_{\mathrm{c}}$ is now determined from the equation

$$
A_{k_{0}}^{\prime}=A_{k_{0}}+4 K_{4} P_{00}^{2}=0,
$$

where $P_{00}$ is the equilibrium value of $P_{0}$ at the transition point. Since at this point $P_{k}=0(k \neq 0), P_{00}$ must satisfy eq. (9) with $K_{1}=K_{1}\left(T_{\mathrm{c}}\right)$. It then follows from (5)-(7), (9), (11) that

$$
\begin{aligned}
E & =\chi_{\mathrm{c}}^{-1} P_{00}\left(1-2 \chi_{\mathrm{c}} K_{4} P_{00}^{2}\right), \\
T_{\mathrm{c}} & =T_{\mathrm{c} 0}-4 C K_{4} P_{00}^{2} .
\end{aligned}
$$

Eq. (12), (13) are parametric equations of the line $T_{\mathrm{c}}(E)$. For

$$
E \ll\left(\chi_{\mathrm{c}}^{3} K_{4}\right)^{-1 / 2}, \quad P_{00} \cong \chi_{\mathrm{c}} E,
$$

and we return to the quadratic dependence

$$
T_{\mathrm{c} 0}-T_{\mathrm{c}}(E) \propto E^{2}
$$

found in our previous work [1].

Slightly below $T_{\mathrm{c}}$, the system is characterized by two parameters, $\psi$ (complex) and $P_{0}$ (real). Putting in (2) $P_{k}=0$ for $k \neq 0, k_{0}$ and $P_{k_{0}}=\psi$, we obtain

$$
\begin{aligned}
F= & \frac{1}{2}\left(K_{1} P_{0}^{2}+K_{4} P_{0}^{4}-2 E P_{0}+\right. \\
& \left.+A_{k_{0}}|\psi|^{2}+4 K_{4} P_{0}^{2}|\psi|^{2}+K_{4}|\psi|^{4}\right) .
\end{aligned}
$$

Here $\psi$ is the order parameter of the phase transition, and $P_{0}$ is a non-critical parameter coupled to $\psi$ through the term $P_{0}^{2}|\psi|^{2}$. To express $F$ as a function of $\psi$ only, we proceed as in a recent work of Benguigui [9]. Solving the equation $\partial F / \partial P_{0}=0$ with respect to $P_{0}$ infinitesimally below $T_{\mathrm{c}}$ and expanding the solution in powers of $|\psi|^{2}$, we get

$$
P_{0}=P_{00}-\frac{4 K_{4} P_{00}|\psi|^{2}}{K_{1}+6 K_{4} P_{00}^{2}}+O\left(|\psi|^{4}\right) \text {. }
$$


Substituting (15) into (14), we arrive at (10), with

$$
B=K_{4} \frac{K_{1}-10 K_{4} P_{00}^{2}}{K_{1}+6 K_{4} P_{00}^{2}} \text {. }
$$

To find the tricritical point, we put $B=0$ and, using eqs. (5) and (11), we obtain

$$
P_{00}^{2}=K_{2}^{2} / 14 K_{3} K_{4}=0.071 / \chi_{c} K_{4} .
$$

Substituting (17) into (12), (13), we get

$$
\begin{aligned}
& E_{\mathrm{t}}=0.228 \chi_{\mathrm{c}}^{-3 / 2} K_{4}^{-1 / 2}, \\
& T_{\mathrm{t}}=T_{\mathrm{c} 0}-0.284 C \chi_{\mathrm{c}}^{-1}
\end{aligned}
$$

(the values obtained by the exact method are $E_{\mathrm{t}}=0.220 \chi_{\mathrm{c}}^{-3 / 2} K_{4}^{-1 / 2}$ and $\left.T_{\mathrm{c} 0}-T_{\mathrm{t}}=0.238 C \chi_{\mathrm{c}}^{-1}\right)$. According to the Landau theory [8], $K_{4}$ is related to the jump in specific heat $\Delta c$ occuring at the smectic $A$ to $C^{*}$ phase transition (at $E=0$ ) by the formula $\Delta c=C^{2} T_{\text {co }} / 4 K_{4}$; hence (we use the exact value for $E_{t}$ )

$$
E_{\mathrm{t}}=0.44\left(\Delta c / C^{2} \chi_{\mathrm{c}}^{3} T_{\mathrm{c} 0}\right)^{1 / 2}
$$

Formula (20) expresses $E_{\mathrm{t}}$ in terms of measurable quantities.

The simplified calculation presented in this paper results in $k_{0}$ independent of $E$ (eq. (4)). According to the more accurate calculation mentioned before, $k_{0}$ is a decreasing function of $P_{00}$, and thereby of $E$. However, for $E \leqslant E_{\mathrm{t}}$, the decrease in $k_{0}$ is negligible, and therefore the above approximation is completely justified. For $E \gg E_{t}$, the growth of $E$ should lead to an appreciable decrease of $k_{0}$ until the helix is completely unwound $[2,3]\left(k_{0}=0\right)$. A theoretical description of this unwinding poses great difficulties, because the Landau theory is inapplicable for first-order transitions far from the tricritical point.

In conclusion, we have demonstrated on the basis of the Landau theory [8], that the line $T_{\mathrm{c}}(E)$ of secondorder phase transitions between the electrically induced uniform smectic $\mathrm{C}$ phase and the electrically distorted smectic $C^{*}$ phase terminates at a tricritical point. Having related the critical quantities $E_{\mathrm{t}}, T_{\mathrm{t}}$ to other measurable properties of the material, we hope to stimulate further experimental work on these interesting liquid crystal phases.

\section{References}

[1] Michelson, A., Benguigui, L. and CaBib, D., To be published in Phys. Rev. A, May 1977.

[2] Meyer, R. B., Liebert, L., Strzelecki, L., Keller, P., $J$. Physique Lett. 36 (1975) L-69.

[3] Martinot-Lagarde, Ph., J. Physique Colloq. 37 (1976) C3-129.

[4] Pieranski, P., Guyon, E. and Keller, P., J. Physique 36 (1975) 1005.
[5] Keller, P., Liebert, L. and Strzelecki, L., J. Physique Colloq. 37 (1976) C3-27.

[6] Priest, R. G., J. Chem. Phys. 65 (1976) 408.

[7] Michelson, A., Phys. Lett. 60A (1977) 29.

[8] Landau, L. D. and Lifshitz, E. M., Statistical Physics (Addison-Wesley Pub. Co.) 1974.

[9] Benguigui, L., Phys. Status Solidi (b) 60 (1973) 835. 